\title{
Segurança do paciente em uma unidade de pronto atendimento: planejamento de ações estratégicas
}

\author{
Patient safety in an emergency care unit: planning strategic actions
}

Seguridad del paciente en una unidad de atención de emergencia: planificación de acciones estratégicas

\author{
Carolina Poite de Siqueira' $\bullet$; Karla Crozeta Figueiredo' ๑; Daiana Kloh Khalaf' $\odot$; \\ Marilene Lowen Wall' ๑; Sayonara de Fátima Faria Barbosall ๑, Thaiane Almeida Silva Pol' ๑ \\ 'Universidade Federal do Paraná, Curitiba, PR, Brasil; "Universidade Federal de Santa Catarina, Florianópolis, SC, Brasil
}

\begin{abstract}
RESUMO
Objetivo: planejar ações estratégicas para a melhoria da qualidade do cuidado e segurança do paciente em Unidade de Pronto Atendimento. Método: pesquisa descritiva, de abordagem qualitativa, desenvolvida em uma Unidade de Pronto Atendimento de um município do sul do Brasil, entre setembro de 2018 e fevereiro de 2019, tendo como participantes os enfermeiros dessa unidade. Utilizou-se como referencial metodológico a Pesquisa Convergente Assistencial, a logicidade do Planejamento Estratégico Situacional e a ferramenta $5 \mathrm{~W} 3 \mathrm{H}$. Foram realizadas oficinas com os participantes, para escolha de problemas na segurança do paciente, proposição de melhorias, adequação e aprovação de planos de ação. Para análise dos dados utilizou-se o software R e grafos de similitude. Resultados: os problemas "comunicação" e "número insuficiente de profissionais" foram escolhidos por 24 enfermeiros, sugerindo cinco propostas, detalhadas em seis planos de ação. Conclusão: o planejamento estratégico situacional permitiu planejar ações estratégicas de melhoria na assistência que são de governabilidade dos enfermeiros.

Descritores: Segurança do Paciente; Cultura Organizacional; Enfermagem em Emergência; Planejamento Estratégico.
\end{abstract}

\section{ABSTRACT}

Objective: to plan strategic actions to improve the quality of care and patient safety in the Emergency Care Unit. Method: this qualitative, descriptive, exploratory study was conducted in an Emergency Care Unit in a municipality in south Brazil, from September 2018 to February 2019, with the unit's nurses as participants. The methodological frame of reference was given by Convergent Care Research, the logic of Situational Strategic Planning, and the 5W3H tool. Workshops were held with the participants to choose problems in patient safety, propose improvements, and adaptation and approval of action plans. Data were analyzed using R software and similarity graphs. Results: nurses chose the problems "communication" and "too few staff" and made five proposals, detailed in six action plans. Conclusion: by situational strategic planning, strategic actions under nurses' governance were planned to improve care.

Descriptors: Patient Safety; Organizational Culture; Emergency Nursing; Strategic Planning.

\section{RESUMEN}

Objetivo: planificar acciones estratégicas para mejorar la calidad de la atención y la seguridad del paciente en la Unidad de Atención de Emergencias. Método: investigación descriptiva, con un enfoque cualitativo, desarrollada en una Unidad de Atención de Emergencias de una ciudad del sur de Brasil, entre septiembre de 2018 y febrero de 2019, cuyas participantes fueron las enfermeras de esta unidad. El marco metodológico utilizado fue la Investigación Convergente de Atención, la lógica de la Planificación Estratégica Situacional y la herramienta 5W3H. Se llevaron a cabo talleres con los participantes para elegir problemas en la seguridad del paciente, proponer mejoras, adecuación y aprobación de planes de acción. El software $\mathrm{R}$ y los gráficos similares se utilizaron para el análisis de datos. Resultados: los problemas de "comunicación" e "número insuficiente de profesionales" fueran elegidos por 24 enfermeras; se sugieren entonces cinco propuestas, detalladas en seis planes de acción. Conclusión: la planificación estratégica situacional permitió planificar acciones estratégicas para mejorar la atención que rigen las enfermeras.

Descriptores: Seguridad del Paciente; Cultura Organizacional; Enfermería de Urgencia; Planificación Estratégica.

\section{INTRODUÇÃO}

Segurança do paciente é definida como a redução de risco de dano desnecessário associado à atenção à saúde até o mínimo aceitável ${ }^{1}$, sendo um tema cada vez mais evidenciado em todo o mundo nos últimos anos. No Brasil, as políticas voltadas para a promoção de uma assistência segura nos estabelecimentos de saúde, com maior ênfase na estruturação de um programa nacional de segurança do paciente datam de 2013.

Como cenário assistencial, as Unidades de Pronto Atendimento (UPA) são organizações complexas pela sua capacidade de articulação e interdependência com diversos pontos na Rede de Atenção à Saúde, de complexidade intermediária, a fim de possibilitar o melhor funcionamento da Rede de Atenção às Urgências² ${ }^{2}$ Os serviços de emergência, como a UPA, caracterizam-se como ambientes estressantes, com alta carga de trabalho cognitivo, ruídos e interrupções frequentes, fatores que favorecem a ocorrência de incidentes ${ }^{3}$, comprometendo, portanto, a segurança do paciente. 
Além disso a alta carga de trabalho, a falta de controle, as falhas de comunicação e de organização, são apontados como fatores que comprometem a segurança do paciente nesse cenário assistencial, sendo que o engajamento dos profissionais no desenvolvimento de procedimentos que aumentem o conhecimento sobre esses fatores, bem como identificar estratégias que possam facilitar a manutenção da segurança do paciente durante os períodos de alta carga de trabalho, são ações para a melhoria do cuidado ${ }^{4}$.

Diante deste contexto, a cultura de segurança é definida como um conjunto de valores, atitudes, competências e comportamentos que buscam a melhoria da qualidade assistencial ${ }^{1}$, é um importante componente estrutural dos serviços, favorecendo a implantação de práticas seguras ${ }^{5}$.

Contudo, alcançá-la é algo complexo, pois a cultura de uma organização está fortemente impregnada nas atitudes e comportamentos das pessoas e, portanto, para mudá-la são necessários o planejamento e a execução de intervenções efetivas que visem a segurança do paciente ${ }^{6}$.

Diante disto, o Planejamento Estratégico Situacional (PES) desenvolvido para resolução de problemas na esfera pública, considera o jogo político, econômico e social que os permeiam, e a percepção da realidade pelos indivíduos (atores) envolvidos nela, que, assim, participam do planejamento ${ }^{7}$.

O PES acontece em quatro momentos: explicativo, normativo, estratégico e tático-operacional, que permitem a seleção de problemas relevantes, planejamento de intervenções, estudo da viabilidade destas e, por fim as colocando em prática; viabilizando aquilo que se planejou ${ }^{7}$, delineando estratégias mais assertivas, podendo ser utilizado como metodologia para o planejamento de melhorias voltadas à segurança do paciente.

Além do uso de uma metodologia, a utilização de ferramentas de planejamento facilita a organização das ações propostas de forma mais clara e objetiva, e sua implementação. Tem-se como exemplos a ferramenta $5 \mathrm{w} 3 \mathrm{~h}$, recurso utilizado para planejar a implementação de ações de forma organizada ${ }^{8}$, e o diagrama de Ishikawa, que organiza as causas ou fatores contribuintes do problema que se quer investigar em grupos ${ }^{9}$.

Desta forma, esta pesquisa teve como objetivo planejar ações estratégicas para a melhoria da qualidade do cuidado e segurança do paciente em uma UPA.

\section{MÉTODO}

Trata-se de um estudo descritivo, de abordagem qualitativa, que utilizou como referencial metodológico a Pesquisa Convergente Assistencial (PCA).

A PCA relaciona o conhecimento teórico à prática profissional, pois prevê que os resultados de pesquisa sejam utilizados nas situações reais de trabalho ${ }^{10}$, permitindo a participação do enfermeiro do local de realização da pesquisa na transformação do seu próprio processo de trabalho, e é desenvolvida em quatro fases: concepção, instrumentação, perscrutação e análise ${ }^{10}$.

A concepção abrange a definição do problema de pesquisa, dos objetivos, da justificativa, revisão de literatura, do referencial metodológico e metodologia, delimitação do espaço físico, escolha dos participantes e de como será procedida a coleta de dados ${ }^{10}$.

O estudo foi desenvolvido em uma UPA de um município do sul do Brasil, teve como critério de inclusão dos participantes ser enfermeiro(a) e trabalhar na UPA, e de exclusão enfermeiro afastado por motivos de saúde, trabalhistas, férias ou indisponíveis no período de coleta de dados.

A coleta de dados ocorreu por meio de quatro oficinas, conduzidas por uma pesquisadora, que é enfermeira assistencial no local da pesquisa, com a presença somente dos enfermeiros participantes, entre setembro de 2018 e fevereiro de 2019, sendo que as falas dos participantes foram gravadas em áudio, após a autorização deles.

A população foi de 31 enfermeiros, com uma amostra final de 24 participantes, sendo que um mesmo enfermeiro participou em mais de uma oficina.

A instrumentação, é delineada pelos "procedimentos mais detalhados" em relação à escolha do espaço físico da pesquisa, dos participantes e dos instrumentos de coleta de dados ${ }^{9}$. Nesta fase obtiveram-se dados mais detalhados acerca do cenário de estudo, assim como foram escolhidos os critérios de inclusão.

A negociação para a realização das oficinas foi realizada primeiramente com a gerência da unidade, em uma breve reunião com o objetivo de explicar a pesquisa, para firmar parcerias e autorização de utilização da estrutura e recursos da UPA. Após foi negociado com os enfermeiros, por meio de um convite para participação nas oficinas por mensagem via aplicativo de celular. 
A perscrutação caracteriza-se como uma "procura minuciosa e profunda de condições para mudanças em todo contexto da investigação" ${ }^{10}$. Esta fase utilizou os resultados obtidos na avaliação da cultura de segurança por meio do questionário Safety Attitudes Questionnaire $(S A Q)^{11}$ previamente realizada no local de pesquisa. Os resultados da cultura de segurança foram apresentados aos enfermeiros na primeira oficina, realizada em cinco encontros, em formato de apresentação de Power Point, para a escolha de dois problemas a serem trabalhados pelos enfermeiros nas próximas oficinas. Cada encontro teve duração média de 40 minutos, teve participação de 14 Enfermeiras, todas do sexo feminino, sendo 12 enfermeiras assistenciais, uma coordenadora e uma supervisora.

Foi pactuado com os enfermeiros que deveriam ser trabalhados problemas de sua governabilidade ou então a construção da viabilidade daqueles que não são de sua governabilidade, assim como propõe a metodologia do PES.

A segunda oficina aconteceu somente em um encontro, e foi discutido o problema "comunicação", teve duração de duas horas. Nessa oficina participaram seis enfermeiras, todas do sexo feminino, sendo três enfermeiras assistenciais, uma coordenadora, uma supervisora e uma administrativa.

Para favorecer a discussão do problema, a fim de encontrar os principais fatores contribuintes associados, foi utilizado um cartaz com o desenho do Diagrama de Ishikawa, facilitando a elaboração de propostas de intervenção.

Para a discussão do problema "Número insuficiente de profissionais", trabalhado durante a terceira oficina, também com somente um encontro, com duração de uma hora, foi utilizada apresentação em Power Point, na qual expuseram-se propostas de como viabilizar o aumento do quadro de enfermagem da UPA. Teve a participação de oito enfermeiras, todas do sexo feminino e assistenciais.

A quarta oficina aconteceu em quatro encontros, com duração média de 40 minutos cada, um em cada turno, com a participação de dez enfermeiros no total, todos do sexo feminino e assistenciais. Nessa oficina, foram apresentados, adequados e aprovados os planos de ação no modelo da ferramenta $5 \mathrm{w} 3 \mathrm{~h}$ elaborados pela pesquisadora na fase de análise, e, após, esses planos foram apresentados para a enfermeira coordenadora da unidade.

As ferramentas $5 w 3 h$ e Diagrama de Ishikawa foram utilizadas nesta pesquisa para planejar as ações estratégicas de melhoria na segurança do paciente na UPA. Cada oficina apresentou um número diferente de participantes, isso se deve a dinâmica do serviço, que nem sempre permite que o enfermeiro se ausente do trabalho, não sendo possível, portanto, que todos os enfermeiros que estavam de plantão pudessem participar das oficinas.

A análise iniciou-se com o processo de apreensão de dados, com a coleta de informações, descrita na fase de perscrutação, por meio das oficinas.

Para organizar os dados, as falas dos participantes foram transcritas no software Word, codificadas com a letra "E" seguida de um número, mantendo, assim a identificação dos participantes anônima, e após, o texto sofreu um pré-processamento, por meio do software $\mathrm{R}$, com a remoção de palavras que não traziam significado (como artigos, pronomes, ou verbos de transição); obtenção do radical de cada termo pelo algoritmo SnowballC; conversão dos radicais novamente à palavra original mais frequente por meio de um algoritmo heurístico; e criação das matrizes através da frequência relativa do termo (tf-prop) e frequência do termo - inverso da frequência no documento (tf-idf).

Para análise das falas, foram elaborados grafos de similitude, e após, determinados os 15 termos mais frequentes, por meio do software R. Os grafos de similitude, são representações gráficas, geradas pelo software R, que "possibilitam identificar as co-ocorrências entre as palavras e seu resultado traz indicações da conexidade entre as palavras, auxiliando na identificação da estrutura da representação" ${ }^{12}$.

Procurou-se nos grafos os termos mais frequentes e quais os termos mais fortemente conectados a eles, e então buscou-se nas falas transcritas quais os assuntos discutidos na presença de tais termos para a compreensão da relação entre eles, buscando, assim, associá-los aos fatores contribuintes apontados pelos enfermeiros, além de buscar novas associações que não apareceram na discussão.

Ao final da análise foi possível a identificação de fatores contribuintes, relacioná-los com as intervenções propostas pelos participantes, e a construção de planos de ação com as intervenções sugeridas para melhoria da segurança do paciente na UPA, utilizando-se a lógica do PES, que possibilitou organizá-las de acordo com seus conceitos e momentos.

Esta pesquisa foi aprovada pelo Comitê de Ética em Pesquisa da instituição. Todos participantes assinaram um Termo de Consentimento Livre e Esclarecido antes de participarem. 


\section{RESULTADOS}

Os participantes dos cinco encontros realizados na primeira oficina escolheram, de forma unânime, os problemas "comunicação" e "número insuficiente de profissionais", somente uma Enfermeira manifestou-se favorável pela escolha também do problema "falta de treinamento de novos profissionais".

A segunda oficina gerou uma base de dados de 643 documentos e 1187 termos. Os 15 termos mais frequentes calculados a partir da frequência relativa na segunda oficina foram: enfermeira, paciente, falado, sabe, coloca, padronização, chega, toda, fica, falta faz, soro, feedback, tudo e entendam. Já a partir da frequência inversa os termos mais frequentes foram: enfermeira, falado, sabe, paciente, padronização, soro, feedback, falta, coloca, chega, toda, computador, fica, rotina e tudo.

As co-ocorrências dos termos a partir da frequência relativa (tf-prop) (Grafo 1) e da frequência inversa (tf-idf) (Grafo 2) podem ser visualizadas a seguir, na Figura 1.

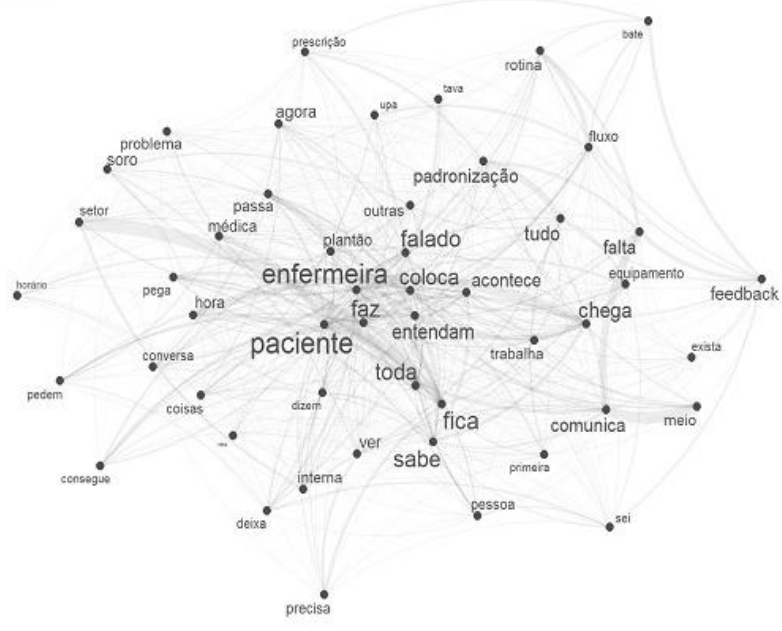

Grafo 1

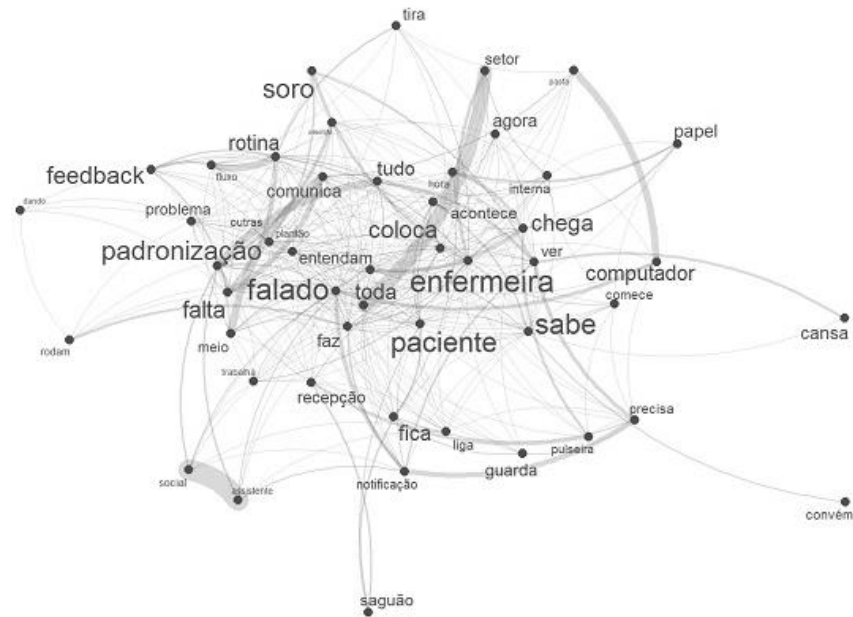

Grafo 2

FIGURA 1: Grafos 1 e 2 da segunda oficina - coocorrência dos termos a partir da frequência relativa dos termos (tf-prop) e da frequência inversa dos termos (tf-idf). Curitiba, Paraná, Brasil, 2019.

Os termos "Enfermeira", "pacientes" e "falado", são visualizados no centro do Grafo 1, conectando-se a muitos outros termos, sendo difícil a visualização de quais termos são mais fortemente relacionados a eles, ou seja, estão relacionados a praticamente tudo, até mesmo pelas altas frequências. O Grafo 2 apresenta os termos mais dispersos e, portanto, possibilita melhor visualização das ligações entre eles.

O Grafo 2 também aponta forte associação entre o termo "falta" e "padronização", "rotina”, "comunicação", e "feedback", reforçando que existe uma lacuna em relação a esses problemas, indicando nós críticos a serem trabalhados.

Os participantes apontaram como intervenções que melhorarão a comunicação na UPA: criação de comissão multiprofissional (CM), reunião participativa, criação de um meio de comunicação oficial, implantação de ações de educação permanente e padronização de fluxos, rotinas e processos de trabalho.

Os enfermeiros consideram que as ações sugeridas poderão melhorar mais de uma causa que prejudica a comunicação. Acreditam que, por exemplo, a participação dos profissionais na elaboração de rotinas e fluxos por meio da CM fará com que se sintam valorizados, melhorando seu comprometimento, assim como a padronização de fluxos e rotinas diminuirá a sobrecarga de trabalho, já que com os processos de trabalho padronizados leva à uma redução nas interrupções geradas por questionamentos originados pela falta de uma linguagem padronizada entre plantões, assim como diminuir falhas nas prescrições médicas e de enfermagem evita o retrabalho. 
A terceira oficina discutiu a proposta para o problema "Número insuficiente de profissionais" e resultou em um corpus com 208 documentos e 463 termos.

A discussão do problema nessa oficina resultou na proposta de implantação do Sistema de Classificação de Pacientes (SCP) para geração de dados que subsidiarão o cálculo de dimensionamento do pessoal de Enfermagem.

Os termos mais frequentes a partir da frequência relativa na terceira oficina foram: faz, fica, todos, sei, plantão, emergente, passa, meses, enfermeira, triagem, paciente, noite, internamento, compromisso, bonitinho. A partir da frequência inversa os 15 termos mais frequentes nesta oficina foram: faz, fica, compromisso, bonitinho, meses, todas, emergente, sei, dizer, triagem, passa, plantão, noite, enfermeira e mensal.

Os Grafos 3 e 4 representam a co-ocorrência dos termos, segundo a frequência relativa e inversa, respectivamente, e são apresentados na Figura 2.

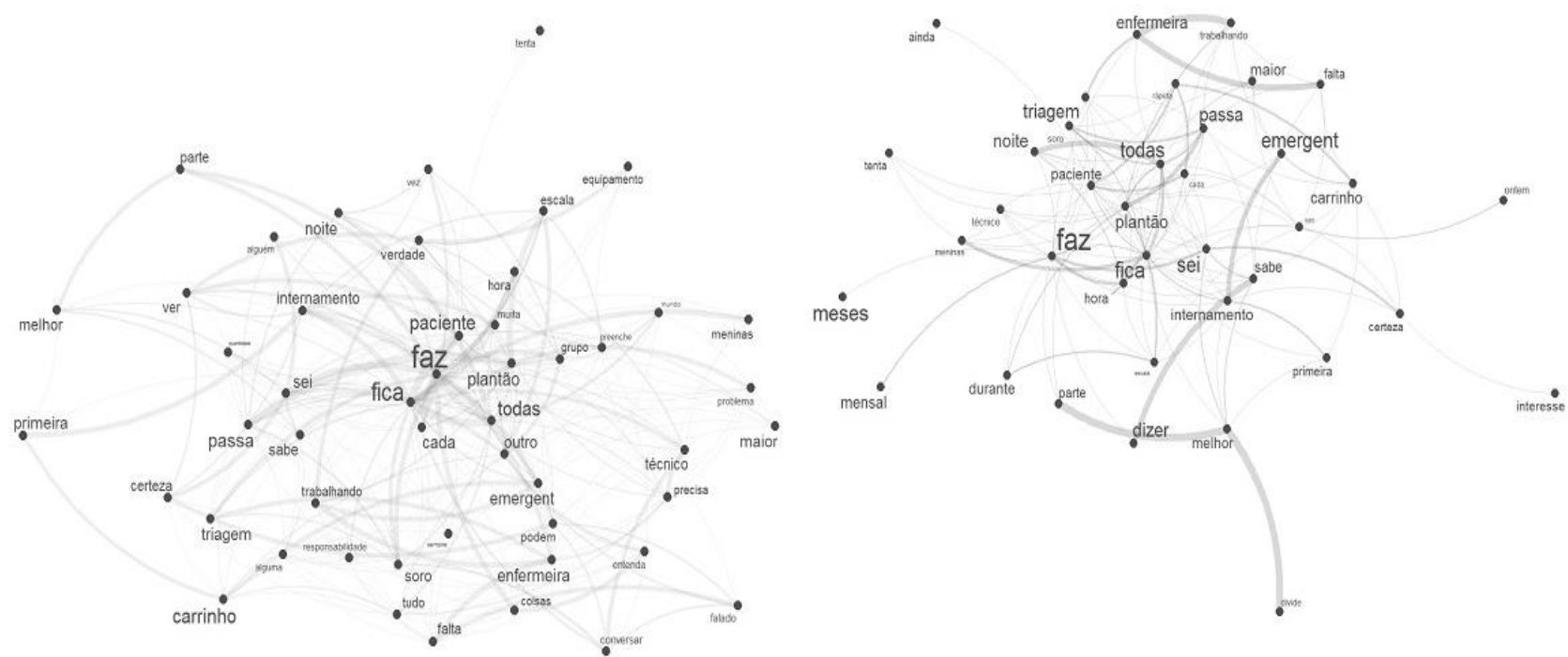

Grafo 3

Grafo 4

FIGURA 2: Grafos 3 e 4 da terceira oficina - co-ocorrência dos termos a partir da frequência relativa dos termos (tf-prop) e da frequência inversa dos termos (tf-idf). Curitiba, Paraná, Brasil, 2019.

Nas oficinas, os enfermeiros não detalharam como executar as ações sugeridas, propuseram as cinco grandes ações, porém, como o modelo 5 w3h prevê o detalhamento baseado em suas questões norteadoras, isto foi desenvolvido pela pesquisadora.

Utilizou-se da logicidade do PES, buscando viabilidade naquilo que se propõe. Elaborou-se, então, os planos de ação fundamentados na análise dos grafos, planos estes adequados e aprovados pelos enfermeiros, nos encontros da quarta oficina.

Assim, foram elaboradas cinco grandes propostas para melhoria da segurança do paciente na UPA: criação de comissão multiprofissional (CM), elaboração e implementação de diretrizes de recursos humanos (RH), padronização de fluxos, rotinas e processos de trabalho, implantação de ações de educação permanente e dimensionamento adequado da equipe de enfermagem.

A Figura 3 apresenta o plano de ação, com as cinco grandes ações propostas pelos enfermeiros durante as oficinas. Cada ação foi detalhada em um plano de ação, que foram entregues à gerência da UPA, as datas de realização foram retiradas pois já estão em andamento e algumas até mesmo finalizadas.

Este plano de ação contém ações planejadas para a realidade pesquisada, porém poderá servir de modelo para outras equipes ou serviços. 


\begin{tabular}{|c|c|c|c|c|c|c|c|}
\hline \multicolumn{8}{|c|}{ AÇÕES DE MELHORIA PARA SEGURANÇA DO PACIENTE NA UPA } \\
\hline $\begin{array}{l}\text { O QUE } \\
\text { (What) }\end{array}$ & $\begin{array}{l}\text { QUEM } \\
\text { (Who) }\end{array}$ & $\begin{array}{l}\text { QUANDO } \\
\text { (When) }\end{array}$ & $\begin{array}{c}\text { ONDE } \\
\text { (Where) }\end{array}$ & $\begin{array}{l}\text { POR QUE } \\
\text { (Why) }\end{array}$ & $\begin{array}{l}\text { COMO } \\
\text { (How) }\end{array}$ & $\begin{array}{c}\text { CUSTOS } \\
\text { (How much) }\end{array}$ & $\begin{array}{c}\text { COMO MEDIR } \\
\text { (How measure) }\end{array}$ \\
\hline Criar a CM & $\begin{array}{l}\text { Coordenação } \\
\text { da UPA } \\
\text { CM } \\
\text { Setor de } \\
\text { Qualidade }\end{array}$ & A definir & $\begin{array}{l}\text { UPA } \\
\text { Afonso } \\
\text { Pena }\end{array}$ & $\begin{array}{l}\text {-Padronizar fluxos, proce ssos de trabalho e } \\
\text { rotinas por meio da participação de } \\
\text { representantes das diversas categorias } \\
\text { profissionais } \\
\text {-Promover a gestão participativa conforme a } \\
\text { Política de Humanização do SUS } \\
\text {-Melhorar a comunicação } \\
\text {-Melhorar o comprometimento dos servidores } \\
\text {-Motivar servidores }\end{array}$ & $\begin{array}{l}\text { - Criar a CM } \\
\text { - Realizar reuniões com a CM a cada } 2 \text { meses }\end{array}$ & $\begin{array}{l}\text { Hora de trabalho dos } \\
\text { profissionais } \\
\text { envolvidos }\end{array}$ & $\begin{array}{l}\text { Conforme plano } \\
\text { de ação } \\
\text { específico }\end{array}$ \\
\hline $\begin{array}{l}\text { Implantar } \\
\text { ações de } \\
\text { Educação } \\
\text { Permanente }\end{array}$ & $\begin{array}{l}\text { Coordenação } \\
\text { da UPA } \\
\text { CM } \\
\text { Setor de } \\
\text { Qualidade } \\
\text { Profissionais } \\
\text { da UPA }\end{array}$ & A definir & $\begin{array}{l}\text { UPA } \\
\text { Afonso } \\
\text { Pena }\end{array}$ & $\begin{array}{l}\text {-Me lhorar a comunicação } \\
\text { - Melhorar a qualidade do atendimento e } \\
\text { consequentemente a segurança do paciente } \\
\text {-Motivar servidores } \\
\text { - Uniform izar conhecimento e práticas } \\
\text { assistencia is } \\
\text {-Facilitar a implantação dos fluxos, rotinas e } \\
\text { processos propostos pe la CM }\end{array}$ & $\begin{array}{l}\text { - Estabe lecer um cronograma anual de } \\
\text { treinamentos de acordo com prioridades } \\
\text { estabe lecidas pe la CM-Solicitar autorização } \\
\text { para pagamento de horas extras aos } \\
\text { profissionais que ministrarão os tre inamentos } \\
\text { - Estabe lecer parceria com o Programa de } \\
\text { Residência Multiprof issional em Urgência e } \\
\text { Emergência da Secretaria Municipal de Saúde } \\
\text { - Realizart treinamentos } \\
\text { - Planejar e implementar o acolhimento de } \\
\text { novos profissionais }\end{array}$ & $\begin{array}{l}\text { Hora de trabalho dos } \\
\text { prof issionais } \\
\text { envolvidos Horas } \\
\text { extras dos profissionais }\end{array}$ & $\begin{array}{l}\text { Conforme plano } \\
\text { de ação } \\
\text { específico }\end{array}$ \\
\hline $\begin{array}{l}\text { Padronizar } \\
\text { fluxos, } \\
\text { rotinase } \\
\text { processos de } \\
\text { trabalho }\end{array}$ & $\begin{array}{l}\text { Coordenação } \\
\text { da UPA } \\
\text { CM } \\
\text { Setor de } \\
\text { Qualidade }\end{array}$ & A definir & $\begin{array}{l}\text { UPA } \\
\text { Afonso } \\
\text { Pena }\end{array}$ & $\begin{array}{l}\text {-Melhorar a comunicação } \\
\text {-Me lhorar a qualidade do atendimento e } \\
\text { consequentemente a segurança do paciente } \\
\text {-Motivar servidor } \\
\text {-Uniform izar práticas assistenciais }\end{array}$ & $\begin{array}{l}\text { - Criar a CM } \\
\text { - Implantar notificação de eventos adversose } \\
\text { near miss } \\
\text { - Plane jar e desenvolver fluxos, rotinas e } \\
\text { processos de acordo com as prioridades } \\
\text { determinadas pe la CM } \\
\text { - Implementar novos fluxos, rotinas e processos } \\
\text { de trabalho }\end{array}$ & $\begin{array}{l}\text { Hora de trabalho dos } \\
\text { profissionais } \\
\text { envolvidos } \\
\text { Horas extras dos } \\
\text { prof issionais para o } \\
\text { plane jamento das } \\
\text { açôes }\end{array}$ & $\begin{array}{l}\text { Conforme plano } \\
\text { de ação } \\
\text { específico }\end{array}$ \\
\hline $\begin{array}{l}\text { Elaborare } \\
\text { Implementar } \\
\text { Diretrizes de } \\
\text { RH }\end{array}$ & $\begin{array}{l}\text { Coordenação } \\
\text { da UPA } \\
\text { CM } \\
\text { Setor de } \\
\text { Qualidade }\end{array}$ & A definir & $\begin{array}{l}\text { UPA } \\
\text { Afonso } \\
\text { Pena }\end{array}$ & $\begin{array}{l}\text {-Padronizar proce dimentos referentes a gestão } \\
\text { de pessoas (escalas, avaliação de desem penho, } \\
\text { absente ísmo etc.) } \\
\text {-Mot ivar servidores } \\
\text {-Estabe lecer feedback adequado }\end{array}$ & $\begin{array}{l}\text { - Criar a CM } \\
\text { - Elaborar as diretrizes de RH da UPA pe la CM } \\
\text { - Implementar as diretrizes de RH da UPA }\end{array}$ & $\begin{array}{l}\text { Hora de trabalho dos } \\
\text { profissionais envolvidos } \\
\text { Horas extras dos } \\
\text { profissionass para o } \\
\text { planejamento das ações }\end{array}$ & $\begin{array}{l}\text { Conforme plano } \\
\text { de ação } \\
\text { especifico }\end{array}$ \\
\hline $\begin{array}{l}\text { Dimensionar } \\
\text { adequadame } \\
\text { nte equipe } \\
\text { de } \\
\text { enfermagem }\end{array}$ & $\begin{array}{l}\text { Coordenação } \\
\text { da UPA } \\
\text { Enferme iros } \\
\text { Assistenciais } \\
\text { da UPA }\end{array}$ & A definir & $\begin{array}{l}\text { UPA } \\
\text { Afonso } \\
\text { Pena }\end{array}$ & $\begin{array}{l}\text {-Determinar se quadro de enfermagem é } \\
\text { adequado ao número de pacientes no setor } \\
\text { internamento } \\
\text {-Obter indicador para ge renciamento de } \\
\text { enfermagem } \\
\text { - Me lhorar a qualidade do atendimento e } \\
\text { consequentemente a se gurança do paciente }\end{array}$ & $\begin{array}{l}\text { - Implantar Sistema de Classificação de } \\
\text { Pacientes (SCP) de Fugulin no setor de } \\
\text { internamento } \\
\text { - Calcular o quadro de pessoal de Enfermagem } \\
\text { da UPA do setor de internamento }\end{array}$ & $\begin{array}{l}\text { Papel, tinta de } \\
\text { impressão } \\
\text { Hora de trabalho dos } \\
\text { prof issionais } \\
\text { envolvidos }\end{array}$ & $\begin{array}{l}\text { Conforme plano } \\
\text { de ação } \\
\text { especifico }\end{array}$ \\
\hline
\end{tabular}

Legenda: CM: comissão Multiprofissional; UPA: Unidade de Pronto Atendimento; SUS: Sistema único de Saúde; RH: Recursos Humanos.

FIGURA 3: Plano de Ação: ações de melhoria para segurança do paciente na UPA. 


\section{DISCUSSÃO}

A CM proposta tem papel central e, portanto, fundamental, uma vez que estará envolvida no desenvolvimento das demais ações. A proposta é que esta comissão seja composta por pelo menos um representante de cada categoria profissional da UPA, permitindo assim, a participação ativa dos trabalhadores da UPA na transformação de seu próprio processo de trabalho, pois discutirão nas reuniões sugestões de melhoria na UPA, e assim, inserindo-os no processo decisório e os corresponsabilizando pelas mudanças. Dessa maneira, a cada reunião os membros da CM definem objetivos a serem alcançados assim como o prazo para finalização das ações propostas.

Existe correlação positiva significativa entre governança compartilhada e engajamento no trabalho, apontando que o envolvimento dos enfermeiros de emergência na tomada de decisões organizacionais e clínicas melhora o envolvimento desses profissionais no trabalho ${ }^{13}$.

No plano de ação para elaboração e implementação de diretrizes de RH, o feedback e a avaliação de desempenho aparecem como formas de operacionalizar o plano de ação. Os achados de outro estudo corroboram com essa ideia, pois os profissionais entrevistados sugeriram a realização de reuniões multiprofissionais "as quais permitam abertura para o diálogo, avaliações de desempenho e feedback contínuos", possibilitando a discussão de problemas do dia a dia, propondo soluções e promovendo uma comunicação mais efetiva ${ }^{14: 5}$.

Outro ponto associado ao problema de comunicação no cenário estudado é a falta de padronização dos processos de trabalho, carecendo de fluxos e rotinas pré-estabelecidos, favorecendo a realização de uma mesma atividade de formas distintas, gerando conflitos entre os profissionais.

Conflitos como esses foram relatados também em outro estudo, no qual profissionais entrevistados apontaram que o não seguimento de normas e regras de trabalho são geradores de conflitos entre equipes e no interior destas. Diante disso, os autores reiteraram que "as regras institucionais buscam organizar e facilitar o processo de trabalho dos enfermeiros e demais profissionais da saúde", mas a participação dos trabalhadores é importante, a fim de os valorizar e corresponsabilizar pelas decisões e implementação das ações ${ }^{15: 265}$.

O ambiente das UPA é geralmente estressante, muitas vezes funcionando acima da sua capacidade de atendimento. Somado a isso, as UPA possuem diversas fragilidades relacionadas à segurança do paciente no processo de trabalho, destacando a não adesão aos protocolos básicos elaborados pelo Ministério da Saúde ${ }^{16}$.

Nesse esforço de mudar a prática para melhorar a segurança do paciente, a padronização de processos de trabalho e implementação de diretrizes de RH permitirá uniformizar as práticas tanto assistenciais como gerenciais, pois a falta de padronização no atendimento permite que profissionais diferentes conduzam atendimentos de formas distintas, 0 que pode levar a um comprometimento da assistência prestada.

Medidas de reorganização de processos de trabalho e práticas profissionais, como a elaboração de protocolos institucionais e educação em serviço, assim como nesta pesquisa, foram sugeridas para melhoria na segurança do paciente ${ }^{17}$.

A implantação de ações de educação permanente visa uniformizar o conhecimento e as práticas assistenciais entre os profissionais, bem como facilitar a implantação dos novos processos de trabalho que a CM proporá.

Uma revisão integrativa encontrou resultados semelhantes a esta pesquisa, apontando que, para alcançar um ambiente assistencial seguro, é preciso realizar educação permanente e melhoria da comunicação ${ }^{18}$.

O segundo problema trabalhado nas oficinas de planejamento de intervenções de melhoria da segurança do paciente foi o número insuficiente de profissionais, para o qual os enfermeiros sugeriram a criação de indicadores que demostrem para a gestão municipal a necessidade de aumento do quadro de pessoal de enfermagem na unidade.

O adequado dimensionamento de pessoal de enfermagem também está associado à mortalidade durante internação hospitalar, com um aumento de $3 \%$ desse risco quando o número de enfermeiros está abaixo do necessário, como concluiu um estudo retrospectivo observacional longitudinal realizado em um hospital inglês ${ }^{19}$.

Calcular o dimensionamento do quadro de pessoal de enfermagem é possível na realidade estudada, conforme pesquisa que utilizou o SCP de Fugulin, possibilitando o cálculo para o adequado dimensionamento, concluindo que havia um déficit de enfermeiros na sala de emergência, apontando também que a maioria dos pacientes se encontrava em cuidados intensivos e semi-intensivos, indicando que o perfil de clientela atendida é semelhante ao de uma UTI ${ }^{16}$.

A classificação de pacientes, além de fornecer dados para o cálculo de dimensionamento do quadro de pessoal de enfermagem, fornecerá indicadores para a melhor sistematização da assistência de enfermagem, pois subsidia a avaliação de quais são os cuidados necessários, configurando-se como ferramenta gerencial e assistencial que, ao utilizar uma linguagem uniforme, auxilia na reestruturação e adequação dos recursos institucionais ${ }^{20}$. 
As discussões realizadas nas oficinas, apontaram, em sua maioria, fatores relacionados à categoria organizacional e, consequentemente, as propostas de melhoria concentraram-se nessa modalidade, com sugestões voltadas a repensar processos já estabelecidos, mas ainda não difundidos igualmente a todos profissionais e tampouco descritos.

\section{Limitações do estudo}

A participação de um reduzido número de enfermeiros durante todas as oficinas apresentou-se como uma limitação do estudo, assim como a especificidade local e o pequeno tamanho da amostra. Portanto, os resultados não são generalizáveis, mas podem servir de base para novas investigações que considerem outros serviços de emergência.

\section{CONCLUSÃO}

A melhoria da segurança do paciente na UPA depende da execução de um conjunto de ações, inter-relacionadas e interdependentes, a serem desenvolvidas por atores sociais. Assim, não será uma medida realizada pela gerência local que resultará em mudanças, mas sim um conjunto de medidas, pensadas por diferentes profissionais com suas diferentes perspectivas, que de meros expectadores passarão a ser agentes ativos de mudança.

Os principais problemas escolhidos pelos enfermeiros - comunicação e número insuficiente de profissionais, bem como as propostas sugeridas para melhoria, apontam o caráter organizacional envolvido nas questões de segurança do paciente na UPA, mostrando a importância do gerenciamento e contribuindo com este.

O esutdo apontou ações estratégicas a serem implementadas, e acredita-se que a padronização de fluxos e rotinas e a criação de diretrizes de RH facilitará a organização do processo de trabalho, bem como da gestão de pessoas, fazendo com que as pessoas sigam um mesmo padrão de condutas profissionais, e, minimizando comportamentos desfavoráveis à segurança do paciente.

Cabe destacar que esta UPA não possui Núcleo de Segurança do Paciente, o que faz os resultados desta pesquisa serem valiosos para o local do estudo.

Conclui-se que as ações de melhoria devem ser planejadas pela gestão desses serviços, a fim de diminuir as lacunas existentes. Para isso, é necessária a busca de quais são os reais problemas que dificultam o desenvolvimento de um ambiente seguro, a fim de elencar as intervenções adequadas e favorecer a chance de sucesso das melhorias implantadas.

\section{REFERÊNCIAS}

1. Raimondi D C., Bernal SCZ., Oliveira JLC, Matsuda LM. Patient safety culture in primary health care: analysis by professional categories. Rev. Gaúcha Enferm. [Internet]. 2019 [cited 2020 Oct 07]; 40(spe):e20180133. DOI: https://doi.org/10.1590/19831447.2019.20180133.

2. Ministério da Saúde (BR). Portaria no 10, de 3 de janeiro de 2017. Redefine as diretrizes de modelo assistencial e financiamento de UPA 24h de Pronto Atendimento como Componente da Rede de Atenção às Urgências, no âmbito do Sistema Único de Saúde. Diário Oficial da União [da] República Federativa do Brasil. 2017 jan 4; (3 Seção 1):34-37.

3. Jones A, Johnstone MJ. Inattentional blindness and failures to rescue the deteriorating patient in critical care, emergency and perioperative settings: Four case scenarios. Aust. Crit. Care [Internet]. 2017 [cited 2020 Apr 13]; 30(4):219-23. DOI: https://doi.org/10.1016/j.aucc.2016.09.005.

4. Källberg AN et al. Physicians' and nurses' perceptions of patient safety risks in the emergency department. International Emergency Nursing [Internet]. 2017 [cited 2020 Feb 23]; 33:14-9. DOI: https://doi.org/10.1016/j.ienj.2017.01.002.

5. Andrade LEL. Patient safety culture in three Brazilian hospitals with different types of management. Cienc. Saúde Colet. [Internet]. 2018 [cited 2020 Apr 13]; 23(1):161-72. DOI: https://doi.org/10.1590/1413-81232018231.24392015-

6. Gutiérrez Ubeda S R. Is an effort needed in order to replace the punitive culture for the sake of patient safety? Revi Calid. Asist. [Internet]. 2016 [cited 2020 Apr 13]; 31(3):173-6. DOI: https://doi.org/10.1016/j.cali.2015.09.007.

7. Silva AK, Sousa JP, Rodrigues W, Cançado AC. Strategic Situational Planning - PES: an bibliometric analysis of Brazilian scientific production. Rev. Serv. Público Brasília [Internet]. 2017 [cited 11 Oct 2020]; 68(2):365-87. DOI: https://doi.org/10.21874/rsp.v68i2.1269.

8. Carlesso F, Tavares RG. Ishikawa diagram and $5 \mathrm{~W} 2 \mathrm{H}$ as quality management tools in clinical analysis laboratory. RBAC [Internet]. 2014 [cited 11 Oct 2020]; 46(1-4):74-9. Available from: http://www.rbac.org.br/wpcontent/uploads/2016/05/RBAC_Vol.46_ns1-4-Completa.pdf

9. Ministério da Saúde (Br). ANVISA. Gestão de Riscos e Investigação de Eventos Adversos Relacionados à Assistência à Saúde. Brasília (BR): Ministério da Saúde; 2017. [cited 2020 Apr 13]. Available from: http://portal.anvisa.gov.br/documents/33852/3507912/Caderno+7++Gestão+de+Riscos+e+Investigação+de+Eventos+Adversos+Relacionados+à+Assistência+à+Saúde/6fa4fa91-c652-4b8b-b56efe466616bd57.

10. Trentini M, Paim L, Silva DMGV. Pesquisa Convergente Assistencial- Delineamento provocador de mudanças nas práticas de saúde. Porto Alegre: Moriá; 2014. 
11. Carvalho REFL, Cassiani SHB. Cross-cultural adaptation of the Safety Attitudes Questionnaire - Short Form 2006 for Brazil. Rev. Lat. Am. Enfermagem [Internet]. 2012 [cited 2018 Jan 03]; 20(3):8 telas. DOI: https://doi.org/10.1590/S010411692012000300020

12. Marchand P, Ratinaud P. L'analyse de similitude appliqueé aux corpus textueles: les primaires socialistes pour l'election présidentielle française. Em: Actes des 11eme Journées internationales d'Analyse statistique des Données Textuelles. JADT [Internet]. 2012 [cited 2020 out 07]; 687-99. Available from: http://lexicometrica.univparis3.fr/jadt/jadt2012/Communications/Marchand,\%20Pascal\%20et\%20al.\%20\%20L'analyse\%20de\%20similitude\%20appliquee\%20aux\%20corpus\%20textuels.pdf .

13. Siller J, Dolansky MA, Clavelle JT, Fitzpatrick JJ. Shared Governance and Work Engagement in Emergency Nurses. J. Emerg. Nurs [Internet]. 2016 [cited 2019 Jul 13]; 42(1):325-30. DOI: https://doi.org/10.1016/j.jen.2016.01.002.

14. Moreira FTLS, Callou RCM, Albuquerque GA, Oliveira RM. Effective communication strategies for managing disruptive behaviors and promoting patient safety. Rev. Gaúcha Enferm. [Internet]. 2019 [cited 2019 Jun 03]; 40(n. esp.):e20180308. DOI: https://doi.org/10.1590/1983-1447.2019.20180308.

15. Amestoy SC, Peixoto RS, Garcia RP, Santos BP, Silva CM, Braga DD. Perception of nurse leaders about managing conflicts in the hospital enviroment. Rev. enferm. UFSM [Internet]. 2016 [cited 2019 Jun 05]; 6(2):259-69. DOI: http://dx.doi.org/10.5902/2179769218167.

16. Paixão DPSS, Batista J, Maziero ECS, Alpendre FT, Amaya MR, Cruz EDA. Adhesion to patient safety protocols in emergency care units. Rev. Bras. Enferm. [Internet]. 2018 [cited 2019 Apr 10]; 71(Suppl 1):622-29. DOI: http://dx.doi.org/10.1590/0034-71672017-0504.

17. Santos PRA, Rocha FLR, Sampaio CSJC. Actions for safety in the prescription, use and administration of medications in emergency care units. Rev. Gaúcha Enferm. [Internet]. 2019 [cited 2019 Jun 03]; 40(n. esp.):e20180347. DOI: https://doi.org/10.1590/1983-1447.2019.20180347.

18. Siman AG, Cunha SGS, Brito MJM. Nursing actions for patient safety in hospitals: integrative review. J. Nurs. UFPE on line [Internet]. 2017 [cited 2019 May 31]; 11(supl.2):1016-24. Available from: https://periodicos.ufpe.br/revistas/revistaenfermagem/article/view/13472/16175.

19. Griffiths P, Maruotti A, Saucedo AR, Redfern OC, Ball JE, Briggs J et al. Nurse staffing, nursing assistants and hospital mortality: retrospective longitudinal cohort study. BMJ Qual. Saf. [Internet]. 2018 [cited 2019 Jun 04]; 28(8):609-17. DOI: http://dx.doi.org/10.1136/bmjqs-2018-008043.

20. Silva KS, Echer IC, Magalhães AMM. Patients dependency degree in relation to the nursing team: a management tool. Esc. Anna Nery [Internet]. 2016 [cited 2019 Jun 04]; 20(3):e20160060. Available from: https://www.scielo.br/scielo.php?pid=S1414$81452016000300205 \&$ script $=$ sci_arttext\&tlng=en . 